\title{
BMJ Open Suffering measurement instruments in palliative care: protocol for a systematic psychometric review
}

Daniel Gutiérrez Sánchez, ${ }^{1,2}$ Rafael Gómez García, ${ }^{1,3}$ Isabel María López-Medina, ${ }^{4}$ Antonio I Cuesta-Vargas ${ }^{2,5,6}$

To cite: Gutiérrez Sánchez D, Gómez García R, López-

Medina IM, et al. Suffering measurement instruments in palliative care: protocol for a systematic psychometric review. BMJ Open 2019;9:e027524. doi:10.1136/ bmjopen-2018-027524

- Prepublication history for this paper is available online. To view these files, please visit the journal online (http://dx.doi. org/10.1136/bmjopen-2018027524).

Received 30 0ctober 2018 Revised 19 February 2019 Accepted 1 March 2019

Check for updates

(C) Author(s) (or their employer(s)) 2019. Re-use permitted under CC BY-NC. No commercial re-use. See rights and permissions. Published by BMJ.

For numbered affiliations see end of article.

Correspondence to Dr Antonio I Cuesta-Vargas; acuesta@uma.es

\section{ABSTRACT}

Introduction The prevention and relief of suffering are regarded as a goal at the end of life; therefore, suffering assessment at the end of life is essential. In this regard, we need instruments that allow us to evaluate this construct for gathering more evidence, as the assessment of suffering is increasingly used in research and the clinical setting. Many measures have been designed to assess this construct, and the selection of the most appropriate instrument is crucial. The aims of this systematic review are to (1) identify the measures assessing suffering in patients with advanced disease and their psychometric properties and (2) evaluate the methodological quality of studies on measurement properties.

Methods and analysis The protocol of this systematic review was developed using the Preferred Reporting Items for Systematic Reviews and Meta-Analyses Protocols Guidelines. A systematic psychometric review of measures assessing suffering in patients with advanced disease and their psychometric properties will be carried out according to the COnsensus-based Standards for the selection of health status Measurement INstruments (COSMIN). The search strategy will be performed following the Peer Review of Electronic Search Strategies. Searches will be conducted in Cumulative Index to Nursing and Allied Health Literature, Medline, PsycINFO, Cochrane Library, SciELO, Open Grey, Scopus, Web of Science and COSMIN database of systematic reviews, and it will be limited by time (1980-2018) and language (only literature in English and Spanish). Literature will be evaluated by two independent reviewers according to the COSMIN checklist, and measurement properties data of each study that meet the inclusion criteria will be scored independently by two researchers according to COSMIN quality ratings.

Ethics and dissemination Ethical approval is not necessary for systematic review protocols. The results will be disseminated by publication in a peer-reviewed journal and presented at a relevant conference.

PROSPERO registration number CRD42018106488.

\section{INTRODUCTION}

Palliative care $(\mathrm{PC})$ is the active holistic care of individuals across all ages with serious health-related suffering due to severe illness, and especially of those near the end of life. WHO defines PC as 'an approach that

\section{Strengths and limitations of this study}

- This will be the most up-to-date psychometric systematic review that identifies, assesses and summarises the evidence regarding suffering assessment instruments.

- The search strategy was designed to be comprehensive, according to the Peer Review of Electronic Search Strategies guidelines.

- This systematic review protocol was developed following the Preferred Reporting Items for Systematic Reviews and Meta-Analyses for Protocols guidelines.

- A limitation of this systematic review is the inclusion of studies published only in English and Spanish, which may lead to language bias.

improves the quality of life of patients and their families facing the problems associated with life-threatening illness through the prevention and relief of suffering by means of early identification and impeccable assessment and treatment of pain and other problems, including physical, psychosocial and spiritual'. ${ }^{1}$ In this context, the prevention and relief of suffering are crucial to the wellbeing and the quality of dying and death of patients and families facing life-threatening diseases. $^{23}$

According to Krikorian and Limonero, suffering is 'a multidimensional and dynamic experience of severe stress that occurs when there is a significant threat to the whole person and regulatory processes are insufficient, leading to exhaustion'. ${ }^{4}$

Suffering assessment is, by definition, subjective, and influenced by physical, psychological, social and spiritual factors that interact with each other. ${ }^{5}$ In this regard, suffering is a difficult construct to measure and one of the most feared symptoms for patients at the end of life, so the assessment of this construct is crucial. ${ }^{7}$

There is a lack of evidence regarding the assessment of suffering in $\mathrm{PC}$, and the 
literature on the measurement of this construct is relatively new. In this regard, we need tools that allow us to measure this construct for gathering more evidence, as the measurement of suffering is increasingly used in research and the clinical setting. ${ }^{8-12}$ In this context, choosing the appropriate measure for assessing suffering is difficult, and the choice is decisive on the accuracy of the results. ${ }^{13}$

Although a previous systematic review to identify the available instruments to assess suffering has been performed, no such review has been published that contrasts the psychometric properties of the instruments assessing this construct in patients with advanced disease according to the most up-to-date COnsensus-based Standards for the selection of health status Measurement INstruments (COSMIN) methodology. ${ }^{814} 15$ Therefore, the aims of this systematic review are to (1) identify the instruments to evaluate suffering in patients with advanced disease and (2) assess the psychometric properties of these instruments.

\section{METHODS \\ Objectives}

This systematic review aims to (1) identify the measures assessing suffering in patients with advanced disease and their psychometric properties and (2) evaluate the methodological quality of studies on psychometric properties.

\section{Design}

The protocol of this systematic review was developed using the Preferred Reporting Items for Systematic Reviews and Meta-Analyses Protocols Guidelines (PRISMA-P), and it was registered in the International Prospective Register of Systematic Reviews. ${ }^{16}$

The systematic review will be carried out according to the most up-to-date COSMIN checklist with a four-point scale. $^{15}$

\section{Search strategy}

The search strategy will be performed following the Peer Review of Electronic Search Strategies (PRESS). ${ }^{17}$ Searches will be conducted in Cumulative Index to Nursing and Allied Health Literature, Medline, PsycINFO, Cochrane Library, SciELO, Open Grey, Scopus, Web of Science and COSMIN database of systematic reviews. The electronic search strategy is shown in table 1 . The search method will be aimed at identifying validation studies of instruments assessing suffering in patients with advanced disease published between 1980 and 2018. Additionally, reference lists of included articles will be checked to identify other relevant literature.

The search strategy will be limited to articles published in English and Spanish.

\section{Inclusion and exclusion criteria}

A study will be included if it meets the following inclusion criteria:
- Includes patients with an advanced disease considered to be a PC population. ${ }^{18}$

- Measures assessing suffering.

- Assesses and describes at least one psychometric property of an instrument according to the COSMIN checklist.

- Reports in English or Spanish and published between January 1980 and August 2018 (inclusive).

We will exclude protocols, addresses, biographies, case reports, comments and editorials, congresses, consensus development conferences, practice guidelines and articles not published in English or Spanish languages.

\section{Outcomes}

All psychometric properties of the instruments assessing suffering will be considered as outcomes in this systematic review. We will adopt the COSMIN terminology and definitions of psychometric properties. ${ }^{15}$

\section{Study screening}

Record duplicates identified by the search strategy will be removed. Titles and abstracts will be screened independently by two review authors. The full article will be retrieved when decisions are unable to be made from abstract and title alone. Any discrepancies will be resolved by discussion (with a third reviewer as necessary). References identified by the search strategy will be entered into Mendeley (Elsevier, Amsterdam) bibliographic software.

The PRISMA flow diagram for this systematic review will be provided.

\section{Quality appraisal}

Full-text articles will be assessed by two review authors independently (DGS and RGG) according to the most up-todate COSMIN checklist with a four-point scale. ${ }^{15}$ This tool considers 10 measurement properties and contains nine boxes with 3-35 items. Each box provides a methodological quality score for instrument development, content validity, structural validity, internal consistency, cross-cultural validity/measurementinvariance, reliability, measurement error, criterion validity, hypotheses testing for construct validity and responsiveness. Each item has four response options: 'very good', 'adequate', 'doubtful' or 'inadequate'. The overall methodological quality score of each psychometric property is obtained by taking the lowest rating of any question in the box. ${ }^{15}$

\section{Data abstraction}

Two researchers independently will participate in the selection of studies and the data extraction process, and any discrepancies will be resolved by discussion. A form will be designed and used to extract and record information for this systematic review. The items of this structured form will include the following general characteristics of each study: study (author and year), population (sample), setting, instrument description, measurement properties, COSMIN score and psychometric values. 
Table 1 Search strategy for Medline

1 ("Suffering”[tiab] OR "distress"[tiab]

2 "Palliative care"[MeSH] OR "terminal care[MeSH]" OR "end of life care"[tiab] "terminally ill" [MeSH] OR "advanced illness"[tiab] OR "terminal illness"[tiab] OR "end of life"[tiab]

3 (Instrument[tiab] OR instruments[tiab] OR measure [tiab] OR measures [tiab] OR questionnaire[tiab] OR questionnaires[tiab] OR scale[tiab] OR scales[tiab] OR tool[tiab] OR tools[tiab] OR survey [tiab] OR test [tiab]

4 Instrumentation[sh] OR methods[sh] OR "Validation Studies"[pt] OR "Comparative Study"[pt] OR "psychometrics"[MeSH] OR psychometr*[tiab] OR clinimetr*[tw] OR clinometr*[tw] OR "outcome assessment [healthcare]"[MeSH] OR "outcome assessment" [tiab] OR "outcome measure " [tw] OR "observer variation"[MeSH] OR "observer variation" [tiab] OR "Health Status Indicators"[Mesh] OR "reproducibility of results"[MeSH] OR reproducib*[tiab] OR "discriminant analysis"[MeSH] OR reliab*[tiab] OR unreliab*[tiab] OR valid*[tiab] OR coefficient[tiab] OR homogeneity[tiab] OR homogeneous[tiab] OR "internal consistency"[tiab] OR (cronbach*[tiab] AND (alpha[tiab] OR alphas[tiab])) OR (item[tiab] AND (correlation*[tiab] OR selection*[tiab] OR reduction*[tiab])) OR agreement[tiab] OR precision[tiab] OR imprecision[tiab] OR "precise values"[tiab] OR test-retest[tiab] OR (test[tiab] AND retest[tiab]) OR (reliab* [tiab] AND (test[tiab] OR retest[tiab])) OR stability[tiab] OR interrater[tiab] OR inter-rater[tiab] OR intrarater[tiab] OR intra-rater[tiab] OR intertester[tiab] OR intertester[tiab] OR intratester[tiab] OR intra-tester[tiab] OR interobserver[tiab] OR inter-observer[tiab] OR intraobserver[tiab] OR intraobserver[tiab] OR intertechnician[tiab] OR inter-technician[tiab] OR intratechnician[tiab] OR intra-technician[tiab] OR interexaminer[tiab] OR inter-examiner[tiab] OR intraexaminer[tiab] OR intra-examiner[tiab] OR interassay[tiab] OR inter-assay[tiab] OR intraassay[tiab] OR intra-assay[tiab] OR interindividual[tiab] OR inter-individual[tiab] OR intraindividual[tiab] OR intra-individual[tiab] OR interparticipant[tiab] OR inter-participant[tiab] OR intraparticipant[tiab] OR intra-participant[tiab] OR kappa[tiab] OR kappa's[tiab] OR kappas[tiab] OR repeatab*[tiab] OR ((replicab*[tiab] OR repeated[tiab]) AND (measure[tiab] OR measures[tiab] OR findings[tiab] OR result[tiab] OR results[tiab] OR test[tiab] OR tests[tiab])) OR generaliza*[tiab] OR generalisa*[tiab] OR concordance[tiab] OR (intraclass[tiab] AND correlation*[tiab]) OR discriminative[tiab] OR "known group"[tiab] OR "factor analysis'[tiab] OR "factor analyses"[tiab] OR dimension*[tiab] OR subscale*[tiab] OR (multitrait[tiab] AND scaling[tiab] AND (analysis[tiab] OR analyses[tiab])) OR "item discriminant"[tiab] OR "interscale correlation*"[tiab] OR error[tiab] OR errors[tiab] OR "individual variability"[tiab] OR (variability[tiab] AND (analysis[tiab] OR values[tiab])) OR (uncertainty[tiab] AND (measurement[tiab] OR measuring[tiab])) OR "standard error of measurement"[tiab] OR sensitiv*[tiab] OR responsive*[tiab] OR ((minimal[tiab] OR minimally[tiab] OR clinical[tiab] OR clinically[tiab]) AND (important[tiab] OR significant[tiab] OR detectable[tiab]) AND (change[tiab] OR difference[tiab])) OR (small*[tiab] AND (real[tiab] OR detectable[tiab]) AND (change[tiab] OR difference[tiab])) OR "meaningful change" [tiab] OR "ceiling effect"[tiab] OR "floor effect"[tiab] OR "Item response model"[tiab] OR IRT[tiab] OR Rasch[tiab] OR "Differential item functioning"[tiab] OR DIF[tiab] OR "computer adaptive testing"[tiab] OR "item bank"[tiab] OR "cross-cultural equivalence"[tiab]

$5 \quad$ \#1 AND \#2 AND \#3 AND \#4

6 "Protocol"[Publication Type] OR "addresses"[Publication Type] OR "biography"[Publication Type] OR "case reports" [Publication Type] OR "comment"[Publication Type] OR "editorial"[Publication Type] OR "congresses" [Publication Type] OR "consensus development conference"[Publication Type] OR "consensus development conference"[Publication Type] OR "practice guideline"[Publication Type]) OR "suffering from"[tiab] OR "animals"[MeSH]

$7 \quad$ \#5 NOT \#6

8 FILTER: Language (English and Spanish)

9 FILTER: Period (1980-2018)

\section{Data synthesis}

Measurement properties data of each study that meets the inclusion criteria will be scored independently by two researchers (DGS and RGG) according to COSMIN quality ratings to identify the most appropriate measure to assess suffering. Any disagreement will be resolved by discussion and consensus, and a third reviewer will be consulted if necessary (AIC-V). In addition, relevant study data will be extracted and reported in the text. A descriptive analysis of the study samples (frequencies and percentages) will be carried out. The correlation between the two reviewer assessments of the main boxes will be calculated using Cohen's Kappa. The quality of evidence for each measurement property will be rated according to the Grading of Recommendations, Assessment, Development and Evaluation approach, adapted for this type of review, into high, moderate, low or very low. ${ }^{19}$ Although we predict that some heterogeneity will be identified in the various assessments of the studies, which will likely make a meta-analysis difficult to apply, where it is possible mean values of statistical analysis (eg, Cronbach's alpha, intraclass correlation coefficient, smallest detectable change, etc) will be calculated from pooled data from methodologically similar studies and the results statistically summarised via meta-analysis into forest plots with estimates of heterogeneity. In addition, sensitivity analysis will be pursued by comparing results from studies with high-quality and low-quality ranking. We will then weight such meta-analyses using both the study's sample size and their quality assessment, as determined by the COSMIN checklist. Statistics of the study samples and inter-rater reliability will be calculated using SPSS V20.0. 


\section{Patient and public involvement}

Patients and the public were not involved in the design of this protocol.

\section{ETHICS AND DISSEMINATION}

Ethical approval is not necessary for systematic review protocols. The results will be disseminated by publication in a peer-reviewed journal and presented at a relevant conference.

\section{DISCUSSION}

Callahan identifies two goals in the field of health, considered of equal category, priority and importance: (1) prevent and cure diseases and (2) help people die in peace. ${ }^{20}$ In this context, it is not only a matter of preserving life at any price but of relieving suffering as much as possible and trying to preserve the life that, in the patient's opinion, deserves to be lived. In this sense, PC plays a fundamental role in relieving the suffering of patients and families. ${ }^{1}$ Thus, the relief of suffering is a major goal to reach in end-of-life patient healthcare, and the measuring of this construct is crucial. ${ }^{21}$ In this context, the main aim of this systematic review is to identify the measures assessing suffering in patients with advanced disease and their psychometric properties and to evaluate the methodological quality of studies on psychometric properties.

\section{Strengths and limitations}

To the best of our knowledge, this will be the most up-todate psychometric systematic review that identifies, assesses and summarises the evidence regarding suffering assessment instruments. This study will permit us to come to a conclusion to allow recommendations about the most desirable measure to evaluate suffering in patients with advanced disease. This review will be carried out following the PRISMA-P, COSMIN and PRESS statements; thus, an appropriate method will be used.

As we limited the search to only studies published in English and Spanish, articles about instruments assessing suffering in patients with advanced disease developed and used in other languages could be missed.

\section{Author affiliations}

${ }^{1}$ Fundación Cudeca, Málaga, Spain

${ }^{2}$ Instituto de Investigación Biomédica de Málaga(IBIMA), Málaga, Spain ${ }^{3}$ GRUPO EE-05 ACPEAL TECH. Instituto de Investigación Biomédica de Málaga (IBIMA), Málaga, Spain

${ }^{4}$ Research Group Nursing and Innovation in Healthcare (CuiDsalud), Department of Nursing, Faculty of Health Sciences, University of Jaén, Jaén, Spain

${ }^{5}$ Department of Physiotherapy, University of Málaga, Málaga, Spain

${ }^{6}$ School of Clinical Sciences, Faculty of Health, Queensland University of Technology, Brisbane, Queensland, Australia

Contributors All authors met the criteria recommended by the International Committee of Medical Journal Editors. All authors made substantial contributions to the conception and design, piloted the inclusion criteria and provided direction on the data extraction and analysis. DGS and AIC-V formulated the idea for the study. DGS drafted the article. RGG and IL-M critically revised the draft for important intellectual content. All authors agreed on the final version.

Funding The authors have not declared a specific grant for this research from any funding agency in the public, commercial or not-for-profit sectors.

Competing interests None declared.

Patient consent for publication Not required.

Provenance and peer review Not commissioned; externally peer reviewed.

Open access This is an open access article distributed in accordance with the Creative Commons Attribution Non Commercial (CC BY-NC 4.0) license, which permits others to distribute, remix, adapt, build upon this work non-commercially, and license their derivative works on different terms, provided the original work is properly cited, appropriate credit is given, any changes made indicated, and the use is non-commercial. See: http://creativecommons.org/licenses/by-nc/4.0/.

\section{REFERENCES}

1. WHO. WHO Definition of Palliative Care. (n.d.). http://www.who.int/ cancer/palliative/definition/en/ (accessed 29 Oct 2017).

2. Cassell EJ. Diagnosing suffering: a perspective. Ann Intern Med 1999;131:531-4.

3. Ferrell B, Connor SR, Cordes A, et al. The national agenda for quality palliative care: the National Consensus Project and the National Quality Forum. J Pain Symptom Manage 2007;33:737-44.

4. Krikorian A, Limonero JT. An integrated view of suffering in palliative care. J Palliat Care 2012;28:41-9.

5. Boston P, Bruce A, Schreiber R. Existential suffering in the palliative care setting: an integrated literature review. J Pain Symptom Manage 2011;41:604-18.

6. Wilson KG, Chochinov HM, McPherson CJ, et al. Suffering with advanced cancer. J Clin Oncol 2007;25:1691-7.

7. den HG. Suffering and dying well: on the proper aim of palliative care. Med Health Care Philos 2017;20:413-24.

8. Krikorian A, Limonero JT, Corey MT. Suffering assessment: a review of available instruments for use in palliative care. $J$ Palliat Med 2013;16:130-42.

9. Adunsky A, Zvi Aminoff B, Arad M, et al. Mini-Suffering State Examination: suffering and survival of end-of-life cancer patients in a hospice setting. Am J Hosp Palliat Care 2007;24:493-8.

10. Beng TS, Ann YH, Guan NC, et al. The suffering pictogram: measuring suffering in palliative care. J Palliat Med 2017;20:869-74.

11. Encarnação P, Oliveira CC, Martins T. Psychometric properties of the suffering assessment questionnaire in adults with chronic diseases or life-threatening illness. Scand J Caring Sci 2018:32:1279-87.

12. Lehmann V, Oerlemans S, van de Poll-Franse LV, et al. Suffering in long-term cancer survivors: an evaluation of the PRISM-R2 in a population-based cohort. Qual Life Res 2011;20:1645-54.

13. Mokkink LB, Prinsen CA, Bouter LM, et al. The COnsensus-based Standards for the selection of health Measurement INstruments (COSMIN) and how to select an outcome measurement instrument. Braz J Phys Ther 2016;20:105-13.

14. Prinsen CAC, Mokkink LB, Bouter LM, et al. COSMIN guideline for systematic reviews of patient-reported outcome measures. Qual Life Res 2018;27:1147-57.

15. Mokkink LB, de Vet HCW, Prinsen CAC, et al. COSMIN Risk of Bias checklist for systematic reviews of Patient-Reported Outcome Measures. Qual Life Res 2018;27:1171-9.

16. Moher D, Shamseer L, Clarke M, et al. Preferred reporting items for systematic review and meta-analysis protocols (PRISMA-P) 2015 statement. Syst Rev 2015;4:1):1.

17. McGowan J, Sampson M, Salzwedel DM, et al. PRESS Peer Review of Electronic Search Strategies: 2015 Guideline Statement. J Clin Epidemiol 2016;75:40-6.

18. McNamara B, Rosenwax LK, Holman CD. A method for defining and estimating the palliative care population. J Pain Symptom Manage 2006;32:5-12.

19. Guyatt GH, Oxman AD, Vist GE, et al. GRADE: an emerging consensus on rating quality of evidence and strength of recommendations. BMJ 2008;336:924-6.

20. Callahan D. Death and the research imperative. N Engl J Med 2000;342:654-6.

21. Hartogh GD. Suffering and dying well: on the proper aim of palliative care. Med Health Care Philos 2017;20:413-24. 